\title{
In the news $\odot$
}

\section{A TOAST TO HEALTH}

Researchers at the National Institutes of Health (NIH) have identified the direct molecular target of resveratrol, a polyphenol found in red wine that mimics the lifespan-extending effects of calorie restriction and potentially protects against diabetes.

Back in 2003, resveratrol was reported to function by directly activating sirtuin 1 (SIRT1), a $\mathrm{NAD}^{+}$-dependent deacetylase that protects against metabolic disease. However, some researchers suggested that this activation may be an experimental artefact. Indeed, Chung and colleagues now report in Cell that resveratrol activates SIRT1 indirectly by inhibiting a class of phosphodiesterases (PDEs) that hydrolyze cyclic AMP.

Previous studies in mice had indicated that AMP-activated protein kinase (AMPK) - which increases $\mathrm{NAD}^{+}$levels and activates SIRT1 - is a key mediator of the effects of resveratrol. The authors now find that cAMP levels increase in skeletal muscle and white adipose tissue in mice following resveratrol administration, and that the observed increase was due to resveratrol inhibiting PDEs. The authors also dissect the biochemical pathway by which cAMP leads to AMPK activation.

"Resveratrol has potential as a therapy for diverse diseases such as type 2 diabetes, Alzheimer's disease and heart disease," commented Chung (NIH News, 2 Feb 2012). However, he cautioned that it is not ready to try as a medicine. It also remains to be seen whether the compound also affects other pathways and, thus, might have side effects. Moreover, it is impossible to consume resveratrol in sufficient quantities to gain any benefit. As commented by Andrew Murray, Cambridge University, UK: "you would need to drink about 700 bottles [of red wine] to get a meaningful dose." (The Telegraph, 2 Feb 2012.) Nevertheless, this study raises hopes for the development of PDE-targeting drugs that would give the same benefits as resveratrol. 